PROCEEDINGS OF THE

AMERICAN MATHEMATICAL SOCIETY

Volume 136, Number 8, August 2008, Pages 2943-2949

S 0002-9939(08)09285-X

Article electronically published on April 15, 2008

\title{
THE GROUND AXIOM IS CONSISTENT WITH V $\neq$ HOD
}

\author{
JOEL DAVID HAMKINS, JONAS REITZ, AND W. HUGH WOODIN
}

(Communicated by Julia Knight)

\begin{abstract}
The Ground Axiom asserts that the universe is not a nontrivial set-forcing extension of any inner model. Despite the apparent second-order nature of this assertion, it is first-order expressible in set theory. The previously known models of the Ground Axiom all satisfy strong forms of $V=$ HOD. In this article, we show that the Ground Axiom is relatively consistent with $V \neq$ HOD. In fact, every model of ZFC has a class-forcing extension that is a model of $\mathrm{ZFC}+\mathrm{GA}+V \neq \mathrm{HOD}$. The method accommodates large cardinals: every model of ZFC with a supercompact cardinal, for example, has a classforcing extension with $\mathrm{ZFC}+\mathrm{GA}+V \neq \mathrm{HOD}$ in which this supercompact cardinal is preserved.
\end{abstract}

The Ground Axiom, introduced by Hamkins and Reitz [10, 9, 4, is the assertion that the universe of set theory is not a nontrivial set-forcing extension of any inner model. That is, the Ground Axiom asserts that if $W$ is an inner model of the universe $V$ and $G$ is $W$-generic for nontrivial forcing, then $W[G] \neq V$. This is true, for example, in the constructible universe $L$, in the model $L\left[0^{\#}\right]$, in the inner model $L[\mu]$ of a measurable cardinal, in most instances of the core model $K$ and in many other canonical models of set theory. Surprisingly, however, the Ground Axiom does not hold in all the canonical inner models, for Schindler has observed that the minimal model $M_{1}$ of one Woodin cardinal is a forcing extension of one of its iterates (see also Theorem 5 below). Precursors to the Ground Axiom include work in [1, where set-genericity over an inner model is considered in the case $L[x]$ for $x$ a real.

Despite the prima facie second order nature of the Ground Axiom assertion-it quantifies, after all, over all inner models of the universe - the Ground Axiom is actually first-order expressible in the language of set theory. This was proved by Reitz [10, 9] and is implicit, independently, in the appendix of Woodin's article 11. These arguments rely, respectively, on recent work of Laver [5], using methods of Hamkins [3, and independent work of Woodin [11, showing that any model of set theory $W$ is first-order definable as a class in all its set-forcing extensions $W[G]$,

Received by the editors February 22, 2007, and, in revised form, June 11, 2007, and June 26, 2007.

2000 Mathematics Subject Classification. Primary 03E35, 03E45, 03E55.

The research of the first author has been supported in part by grants from the CUNY Research Foundation and from the Netherlands Organization for Scientific Research.

The research of the third author has been supported in part by the National Science Foundation.

We note that the authors of this article constitute three mathematical generations: Reitz was a dissertation student of Hamkins, who was a dissertation student of Woodin.

(C)2008 American Mathematical Society Reverts to public domain 28 years from publication 
using parameters in $W$. Because the definition is uniform, one can effectively quantify over the possible ground models of $V$ by quantifying over the possible parameters to be used in this definition. Reitz [10, 9] identifies the first-order properties of a parameter that allow it to succeed in defining a ground model.

While the Ground Axiom fails, of course, after any nontrivial set forcing, Reitz observed that it can hold after certain nontrivial class forcing iterations. In an extreme instance of this, Friedman 2 produces a real $R$, class-generic but not set-generic over $L$, such that there are no models strictly intermediate between $L$ and $L[R]$. Another general form of the phenomenon can be obtained via work of McAloon [7] and others, who showed long ago how to force strong versions of $V=$ HOD by coding the universe into various combinatorial features. One method of doing so is to force the GCH to hold or fail at successive cardinals, coding one bit of information each time, so that in the resulting forcing extension every set of ordinals is explicitly coded in the GCH pattern. Reitz observed that this strong form of $V=$ HOD implies the Ground Axiom: if every set in $V$ is coded into the continuum function and $V=W[h]$ for some inner model $W$ and set forcing $h \subseteq \mathbb{Q} \in W$, then since $V$ and $W$ agree on the continuum function above the size of the forcing $\mathbb{Q}$, it follows that every set in $V$ is already coded in $W$, and so $W=V$ and the forcing $h$ was trivial. This argument shows that the Ground Axiom is consistent with the failure of the GCH. Further, since the forcing to code sets into the continuum function could be delayed to start above any given cardinal, Reitz's argument shows that the Ground Axiom is consistent with any possible behavior in any fixed $V_{\lambda}$. Thus, the Ground Axiom does not provably imply any of the structural regularities of the canonical models $L, L[\mu]$ and so on, in which it holds.

Because all of the previously known models of the Ground Axiom have satisfied various strong forms of $V=\mathrm{HOD}$, one naturally inquires whether the Ground Axiom is consistent with $V \neq$ HOD. This question is answered affirmatively by the main theorem of this article.

Main Theorem 1. If ZFC is consistent, then it is consistent with the Ground Axiom plus $V \neq \mathrm{HOD}$.

This theorem is a consequence of the more specific Theorem 2 below, which will be generalized in Theorem 3 . We conclude that the Ground Axiom and the hypothesis $V=\mathrm{HOD}$ are mutually independent, since all four combinations are possible: (1) both hold in $L$; (2) both fail in the extension $L[c]$ to add a Cohen real; (3) $\neg \mathrm{GA}+V=\mathrm{HOD}$ holds in various set-forcing extensions of $L$; and finally, (4) $\mathrm{GA}+V \neq$ HOD holds in the models of this article.

Theorem 2. The constructible universe $L$ has a class-forcing extension, preserving cardinals and cofinalities, satisfying $\mathrm{ZFC}+\mathrm{GA}+\mathrm{GCH}+V \neq H O D$.

Proof. For any regular cardinal $\gamma$, let $\operatorname{Add}(\gamma, 1)$ be the forcing notion to add a Cohen subset to $\gamma$ by initial segment, that is, the poset of binary sequences ${ }^{<\gamma} 2$ ordered by end extension. In $L$, let $\mathbb{P}$ be the (reverse) Easton support class iteration that forces at each regular cardinal stage $\gamma$ with $\operatorname{Add}(\gamma, 1)$, and suppose that $G \subseteq \mathbb{P}$ is $L$-generic. We will show that $L[G]$ satisfies $\mathrm{ZFC}+\mathrm{GA}+\mathrm{GCH}+V \neq \mathrm{HOD}$.

The fact that the class-forcing extension $L[G]$ preserves all cardinals and cofinalities and satisfies $\mathrm{ZFC}+\mathrm{GCH}$ is a standard argument, whose details we leave to the reader. The argument is assisted by the fact that for any cardinal $\kappa$ the forcing $\mathbb{P}$ factors as $\mathbb{P}_{1} * \dot{\mathbb{P}}_{2}$, where $\mathbb{P}_{1}$ is the forcing up to stage $\kappa$ and $\dot{\mathbb{P}}_{2}$ is the (name of the) 
forcing at stages $\kappa$ and beyond. Since $\dot{\mathbb{P}}_{2}$ is forced to be $<\kappa$-closed, it follows that all sets of hereditary size less than $\kappa$ in the full extension $L[G]$ are in the set-forcing extension $L\left[G_{1}\right]$ corresponding to the first factor $\mathbb{P}_{1}$, and this is a model of ZFC.

The fact that $L[G] \models V \neq$ HOD is also a standard argument, a consequence of the fact that $\mathbb{P}$ is nontrivial and almost homogeneous. Let us sketch the details. A partial order $\mathbb{Q}$ is almost homogeneous if for any two conditions $p, q \in \mathbb{Q}$ there is an automorphism $\pi$ of $\mathbb{Q}$ such that $\pi(p)$ is compatible with $q$. Any automorphism $\pi$ of $\mathbb{Q}$ induces an automorphism of the universe $V^{\mathbb{Q}}$ of $\mathbb{Q}$-names $\tau$ by recursively applying $\pi$ inside the names, namely, $\tau^{\pi}=\left\{\left\langle\sigma^{\pi}, \pi(p)\right\rangle \mid\langle\sigma, p\rangle \in \tau\right\}$. The automorphism $\pi$ extends uniquely to an automorphism $\pi$ of the corresponding Boolean algebra $\mathrm{RO}(\mathbb{Q})$, and applying this to the Boolean values of statements in the forcing language, one easily proves by induction on formulas that

$$
\pi\left(\llbracket \varphi\left(\tau_{0}, \ldots, \tau_{n}\right) \rrbracket\right)=\llbracket \varphi\left(\tau_{0}^{\pi}, \ldots, \tau_{n}^{\pi}\right) \rrbracket .
$$

Since $\pi\left(1^{\mathbb{Q}}\right)=1^{\mathbb{Q}}$, it follows that $\check{x}^{\pi}=\check{x}$ for any $x$ in the ground model $V$, and so the Boolean value $\llbracket\left(\check{x}_{0}, \ldots, \check{x}_{n}\right) \rrbracket$ is fixed by any such $\pi$. Since for any conditions $p$ and $q$ we may find by almost homogeneity an automorphism $\pi$ such that $\pi(p)$ is compatible with $q$, it follows that the only Boolean values fixed by all automorphisms $\pi$ are 0 and 1 . Thus, for any parameters $x_{i}$ from the ground model, $\varphi\left(x_{0}, \ldots, x_{n}\right)$ holds in the extension if and only if $\llbracket \varphi\left(\check{x}_{0}, \ldots, \check{x}_{n}\right) \rrbracket=1$ in the ground model. In particular, for any ordinal parameters $\beta, \alpha_{0}, \ldots, \alpha_{n}$, the definable set $\left\{\alpha<\beta \mid \varphi\left(\alpha, \alpha_{0}, \ldots, \alpha_{n}\right)\right\}$ in the forcing extension $V^{\mathbb{Q}}$ is definable in the ground model $V$ by $\left\{\alpha<\beta \mid \llbracket \varphi\left(\check{\alpha}, \check{\alpha}_{0}, \ldots, \check{\alpha}_{n}\right) \rrbracket=1\right\}$. Thus, any ordinal-definable set of ordinals in the forcing extension was already definable in the ground model, and so if $\mathbb{Q}$ adds any new sets at all, it will force $V \neq$ HOD.

In our case, the poset $\mathbb{Q}=\operatorname{Add}(\gamma, 1)$ is easily seen to be almost homogeneous, because if $p, q \in 2^{<\gamma}$, then there is an automorphism $\pi$ of $\mathbb{Q}$ that simply flips bits in the binary sequences in order to ensure that $\pi(p)$ is compatible with $q$. Iterating this, we see that every individual stage of forcing in the iteration $\mathbb{P}$ is (forced to be) almost homogenous. Further, for any two conditions $p, q \in \mathbb{P}$ in the full iteration, one may combine the various names for these bit-flipping automorphisms at each stage of forcing to construct a grand automorphism $\pi: \mathbb{P} \rightarrow \mathbb{P}$ of the whole iteration such that $\pi(p)$ is compatible with $q$. So our iteration $\mathbb{P}$ is nontrivial and almost homogeneous, and consequently $L[G] \models V \neq$ HOD, as we claimed.

Finally, in the new part of our argument, we prove that the Ground Axiom holds in $L[G]$. Suppose to the contrary that GA fails there. This means that $L[G]$ is a nontrivial set-forcing extension of some inner model $W$. That is, $L[G]=W[h]$ for some nontrivial $W$-generic set forcing $h \subseteq \mathbb{Q} \in W$. We may assume that the underlying set of $\mathbb{Q}$ is a cardinal in $W$. Let $\kappa$ be a singular strong limit cardinal of $W$ with $\kappa>|\mathbb{Q}|^{W}$, and factor the forcing $\mathbb{P}$ as $\mathbb{P}_{1} * \dot{\mathbb{P}}_{2}$, where $\mathbb{P}_{1}$ is the forcing up to stage $\kappa$ and $\dot{\mathbb{P}}_{2}$ is the forcing from stage $\kappa$ onwards. Since $\kappa$ is singular in $W$, it remains singular in $W[h]=L[G]$ and hence was singular in $L$. So the stage $\kappa$ forcing of $\mathbb{P}$ is trivial. The stages of forcing beyond $\kappa$ are $\leq \kappa$-closed, and so $\mathbb{P}_{1} \Vdash \dot{\mathbb{P}}_{2}$ is $\leq \kappa$-closed. We similarly factor the generic filter as $G=G_{1} * G_{2} \subseteq \mathbb{P}_{1} * \dot{\mathbb{P}}_{2}$ and the extension as $L[G]=L\left[G_{1}\right]\left[G_{2}\right]$. Since $G_{1} \in W[h]$, there is a $\mathbb{Q}$-name $\dot{G}_{1} \in W$ such that $G_{1}=\left(\dot{G}_{1}\right)_{h}$. Since $|\mathbb{Q}|<\kappa$ and $G_{1}$ is determined by the $\kappa$-sequence of subsets added at each stage of forcing, we may find a name $\dot{G}_{1}$ of hereditary size $\kappa$. Since also $\left(2^{<\kappa}\right)^{W}=\kappa$, as $\kappa$ is a strong limit in $W$, we may find in $W$ a set $A \subseteq \kappa$ such 
that both $\dot{G}_{1}$ and the binary sequence space $\left({ }^{<\kappa} 2\right)^{W}$ are coded by $A$. Since $A \in W$, it follows that $L[A] \subseteq W$. By the choice of $A$, we have arranged that $\dot{G}_{1} \in L[A]$ and $\left({ }^{<\kappa} 2\right)^{L[A]}=\left({ }^{<\kappa} 2\right)^{W}$. In particular, $\mathbb{Q} \in L[A]$ and $h \subseteq \mathbb{Q}$ is $L[A]$ generic, so we may consider the forcing extension $L[A][h]$. Both $A$ and $h$ are subsets of $\kappa$ in the full extension $L\left[G_{1}\right]\left[G_{2}\right]$, and since the second factor of this forcing is $\leq \kappa$-closed, it follows that $A$ and $h$ are in $L\left[G_{1}\right]$, and consequently $L[A][h] \subseteq L\left[G_{1}\right]$. Conversely, $G_{1}$ is in $L[A][h]$ since $\dot{G}_{1} \in L[A]$ and $G_{1}=\left(\dot{G}_{1}\right)_{h}$. We therefore conclude that $L[A][h]=L\left[G_{1}\right]$. We next aim to prove that $W=L[A]$, from which we will derive a contradiction. This key step makes use of the following definitions and results.

Definition 2.1 ([3]). Suppose that $W \subseteq V$ are both transitive models of (a suitable small fragment of) ZFC and $\delta$ is a cardinal in $V$.

(1) The extension $W \subseteq V$ exhibits the $\delta$ cover property if for each $A \in V$ with $A \subseteq W$ and $|A|^{V}<\delta$ there is a $B \in W$ such that $A \subseteq B$ and $|B|^{W}<\delta$.

(2) The extension $W \subseteq V$ exhibits the $\delta$ approximation property if whenever $A \in V$ with $A \subseteq W$ and $A \cap B \in W$ for all $B \in W$ with $|B|^{W}<\delta$, then $A \in W$.

Lemma 2.2 ([3]). If $V \subset V[G]$ is a forcing extension by forcing of the form $\mathbb{Q}_{1} * \dot{\mathbb{Q}}_{2}$, where $\mathbb{Q}_{1}$ is nontrivial and $\Vdash \dot{\mathbb{Q}}_{2}$ is $\leq\left|\overleftarrow{\mathbb{Q}}_{1}\right|$ strategically closed, then $V \subseteq V[G]$ satisfies the $\delta$ cover and $\delta$ approximation properties for $\delta=\left|\mathbb{Q}_{1}\right|^{+}$.

Lemma 2.3 (Hamkins, Laver [5]). Suppose that $W, W^{\prime}$ and $V$ are all transitive models of ZFC and that $\delta$ is a regular cardinal of $V$. Suppose further that $W \subseteq V$ and $W^{\prime} \subseteq V$, that these extensions both exhibit the $\delta$ cover and approximation properties, and that $\mathcal{P}(\delta)^{W}=\mathcal{P}(\delta)^{W^{\prime}}$ and $\left(\delta^{+}\right)^{W}=\left(\delta^{+}\right)^{W^{\prime}}=\left(\delta^{+}\right)^{V}$. Then $W=$ $W^{\prime}$.

An alternative proof of Lemma 2.2 is provided by [8]. In Lemma 2.2, the second factor $\mathbb{Q}_{2}$ may be trivial. Laver's original version of Lemma 2.3 applied only to small forcing, which Hamkins improved to extensions as above with the $\delta$ cover and approximation properties. An analogous version of the lemma for small forcing was proved independently by Woodin in [11, Lemma 21].

Returning to the proof of Theorem 2, we now argue that $W=L[A]$. We will show that the models $W$ and $L[A]$, contained in $L[G]$, satisfy the hypotheses of Lemma 2.3, and hence are equal. Specifically, since $L[A][h]=L\left[G_{1}\right]$, it follows that $G_{2}$ is $L[A][h]$-generic for $\mathbb{P}_{2}$ and $L[G]=L\left[G_{1}\right]\left[G_{2}\right]=L[A][h]\left[G_{2}\right]$. In particular, $L[G]=L[A][h]\left[G_{2}\right]$ is a forcing extension of $L[A]$ by the forcing $h * G_{2} \subseteq \mathbb{Q} * \dot{\mathbb{P}}_{2}$, using a suitable $\mathbb{Q}$-name for $\mathbb{P}_{2}$. Let $\delta=|\mathbb{Q}|^{+}$, computed in $L[A]$, which agrees with $W$ on this calculation. Since $\mathbb{Q}$ is nontrivial and $\mathbb{P}_{2}$ is $\leq \kappa$-closed in $L[A][h]=$ $L\left[G_{1}\right]$, it follows by Lemma 2.2 that $L[A] \subseteq L[A][h]\left[G_{2}\right]$ exhibits the $\delta$ cover and approximation properties. The extension $W \subseteq W[h]$ also satisfies the $\delta$ cover and approximation properties, since $\delta=|\mathbb{Q}|^{+}$in $W$ and Lemma 2.2 allows that the second factor $\mathbb{Q}_{2}$ is trivial. In both cases, the larger model is the same ultimate forcing extension $L[A][h]\left[G_{2}\right]=L[G]=W[h]$. Because $W$ and $L[A]$ have the same bounded subsets of $\kappa$, we know $P(\delta)^{W}=P(\delta)^{L[A]}$, and all three models $W, L[A]$, and $L[G]$ agree on $\delta^{+}$. By Lemma 2.3 , therefore, we conclude that $W=L[A]$. This implies that $W[h]=L[A][h]=L\left[G_{1}\right]$, contradicting the assumption that $W[h]=L\left[G_{1}\right]\left[G_{2}\right]$. 
The proof of Theorem 2 is flexible and generalizes in a variety of ways. For example, not much was used about the specific iteration $\mathbb{P}$. For establishing GA, we needed to know only that the stage $\gamma$ forcing was $<\gamma$-closed, and we could easily have accommodated $\operatorname{Add}\left(\gamma, \gamma^{++}\right)$or occasionally $\operatorname{Coll}\left(\gamma, \gamma^{+}\right)$, for example, without any difficulty in the argument. Also, we needn't have forced specifically at every regular cardinal stage $\gamma$, but could have forced at regular cardinals in some other unbounded pattern. Thus, the argument establishes that after forcing over $L$ with any of the usual reverse Easton iterations of closed forcing, one obtains the Ground Axiom in the extension.

We now extend Theorem 2 beyond $L$.

Theorem 3. Every model of ZFC has a class-forcing extension satisfying the Ground Axiom plus $V \neq \mathrm{HOD}$.

Proof. We will follow the proof of Theorem 2, forcing over $V$ instead of $L$, but modified with a suitable preparatory forcing. A key property of $L$ used in the proof of Theorem [2 was that when we had $L[G]=W[h]$, we were freely able to deduce that $L \subseteq W$. In the general case, we force over $V$ to obtain $V[G]=W[h]$, and we will similarly want to deduce $V \subseteq W$. In general, however, this does not follow. But we can ensure the conclusion in our case by means of suitable preparatory forcing. Specifically, we shall first force a strong version of $V=$ HOD, asserting that every set of ordinals is coded explicitly into the continuum function. This can be accomplished, as we mentioned earlier, with a class-forcing iteration, forcing the GCH to hold or fail at successive cardinals so as to code one bit of information at each stage of forcing, either by means of a suitable bookkeeping function, or more elegantly, by letting the generic filter decide at each stage how to code (an idea due to Sy Friedman). The general idea is due originally to McAloon [7, and coding specifically with GCH has been used many times in the set-theoretical literature; see 10, 9 for a recent detailed account of it in connection with the Ground Axiom. For our purposes, it is convenient to perform the coding only at successor cardinals, whose power sets are more easily controlled. In this way, we find an extension $\bar{V}$ of $V$ satisfying the Continuum Coding Axiom:

For every set of ordinals $x \subset \alpha \in \mathrm{ORD}$, there exist arbitrarily large regular $\theta$ such that $\forall \beta<\alpha \quad \beta \in x \Longleftrightarrow 2^{\aleph_{\theta+\beta+1}}=\aleph_{\theta+\beta+2}$.

Working now in $\bar{V}$, let $\mathbb{P}$ be the reverse Easton iteration forcing with $\operatorname{Add}(\gamma, 1)$ at each regular cardinal stage $\gamma$ for which $2^{<\gamma}=\gamma$, and suppose $G$ is $\bar{V}$-generic for $\mathbb{P}$. The Continuum Coding Axiom ensures that the GCH often holds at successor cardinals, and so such stages occur unboundedly often in the ordinals. Just as in Theorem 2, we know that $\bar{V}[G] \models \mathrm{ZFC}+V \neq \mathrm{HOD}$ and also that $\bar{V}[G]$ has the same cardinals and the same continuum function as $\bar{V}$. In $\bar{V}[G]$, therefore, every set of ordinals in $\bar{V}$ is coded into the continuum function in $\bar{V}[G]$ in the manner of the Continuum Coding Axiom.

We complete the proof by showing that $\bar{V}[G]$ satisfies the Ground Axiom. Suppose to the contrary that $\bar{V}[G]=W[h]$, for some transitive inner model $W$, where $h$ is $W$-generic for some forcing notion $\mathbb{Q} \in W$. Since this is set-forcing over $W$, it follows that $W$ and $W[h]$ have the same cardinals and continuum function above $|\mathbb{Q}|$. Since every set of ordinals in $\bar{V}$ is coded into this part of the continuum function, in common between $W$ and $\bar{V}[G]$, it follows that every set of ordinals in $\bar{V}$ is coded in $W$, and consequently $\bar{V} \subseteq W$. Next, as in Theorem 2, choose 
a singular strong limit $\kappa>|\mathbb{Q}|$ and factor the forcing $\mathbb{P}=\mathbb{P}_{1} * \dot{\mathbb{P}}_{2}$ at stage $\kappa$, giving $\bar{V}[G]=\bar{V}\left[G_{1}\right]\left[G_{2}\right]$. Once again we can find $A \subseteq \kappa$ with $A \in W$ such that $A$ codes both $\left({ }^{<\kappa} 2\right)^{W}$ and $\dot{G}_{1}$, a $\mathbb{Q}$-name for $G_{1}$. As in Theorem 2, we conclude $\left({ }^{<\kappa} 2\right)^{\bar{V}}[A]=\left({ }^{<\kappa} 2\right)^{W}$, using the fact that $\bar{V} \subset W$ for the forward inclusion. Thus, $h$ is $\bar{V}[A]$-generic for $\mathbb{Q}$, and $\bar{V}[A][h]=V\left[G_{1}\right]$. If $\delta=|\mathbb{Q}|^{+}$computed in $\bar{V}[A]$ or $W$, which agree, then the two extensions $\bar{V}[A] \subset \bar{V}[A][h]\left[G_{2}\right]$ and $W \subset W[h]$ both satisfy the $\delta$ cover and approximation properties. Since $\bar{V}[A]$ and $W$ have the same $\mathcal{P}(\delta)$ and the three models $\bar{V}[A], W$ and $\bar{V}[G]$ all have the same $\delta^{+}$, we conclude again by Lemma 2.3 that $\bar{V}[A]=W$. This implies $W[h]=\bar{V}[A][h]=\bar{V}\left[G_{1}\right]$, contradicting $W[h]=\bar{V}\left[G_{1}\right]\left[G_{2}\right]$. So $\bar{V}[G]$ satisfies the Ground Axiom.

The argument is flexible enough to accommodate large cardinals, by simply starting the coding forcing at a sufficiently high level. To demonstrate, we explain the situation below with supercompact cardinals.

Corollary 4. If $\kappa$ is supercompact in $V$, then there is a class-forcing extension preserving the supercompactness of $\kappa$ and satisfying $\mathrm{GA}+V \neq \mathrm{HOD}$.

Proof. Suppose $\kappa$ is a supercompact cardinal in $V$. Perform the Laver preparation of $\kappa$ (see [6] to obtain a model $V\left[G_{0}\right]$ where the supercompactness of $\kappa$ is indestructible by $<\kappa$-directed closed forcing). Next, perform the forcing to code the universe into the continuum function, but delay the start of the coding until beyond stage $\kappa$, to obtain an extension $V\left[G_{0}\right] \subseteq \bar{V}$ in which every set of ordinals is coded explicitly into the continuum function as in Theorem 3 . Since the coding forcing is $<\kappa$-directed closed (and since it factors into set forcing followed by highly closed forcing), it follows that $\kappa$ remains supercompact and Laver indestructible in $\bar{V}$. Finally, let $\mathbb{P}$ be the reverse Easton iteration as in Theorem 3 but delay the start of the forcing until beyond $\kappa$. If $G \subseteq \mathbb{P}$ is $\bar{V}$-generic, then the proof of Theorem 3 shows that $\bar{V}[G]$ exhibits the Ground Axiom plus $V \neq$ HOD. The supercompactness of $\kappa$ is preserved to $\bar{V}[G]$, in the usual Laver argument, because $\mathbb{P}$ is $<\kappa$-directed closed and factors at arbitrarily high levels as set forcing followed by highly closed forcing.

Alternatively, a more streamlined argument would simply use Friedman's method of forcing $V=\mathrm{HOD}$, letting the generic filter decide at each stage whether to force GCH there or not, followed by the iteration $\mathbb{P}$; the usual arguments show that this combined forcing essentially preserves all large cardinals, while forcing $V \neq \mathrm{HOD}$, and our argument shows that it also forces GA.

Lastly, we close the paper by mentioning without proof a result observed by the third author.

Theorem 5. Suppose that $M$ is an $\left(\omega_{1}+1\right)$-iterable countable Mitchell-Steel model and $M \models \mathrm{ZFC}+$ "There is a Woodin cardinal". Then there exists $N \subset M$ such that

(1) $M$ is a nontrivial set generic extension of $N$,

(2) $N \models \mathrm{ZFC}+$ "There is a Woodin cardinal," and

(3) $N$ is an $\left(\omega_{1}+1\right)$-iterable Mitchell-Steel model.

Such models, therefore, do not satisfy the Ground Axiom. 


\section{REFERENCES}

1. A. Beller, R. Jensen, and P. Welch, Coding the Universe, London Mathematical Society Lecture Note Series, Cambridge University Press, 1982. MR645538 (84b:03002)

2. Sy D. Friedman, Fine Structure and Class Forcing, vol. 3 of Series in Logic and Its Applications, de Gruyter, 2000. MR.1780138 (2001g:03001)

3. Joel David Hamkins, Extensions with the approximation and cover properties have no new large cardinals, Fundamenta Mathematicae 180 (2003), no. 3, 257-277. MR2063629 (2005m:03100)

4. - The Ground Axiom, Oberwolfach Report 55 (2005), 3160-3162.

5. Richard Laver, Certain very large cardinals are not created in small forcing extensions, to appear in the Annals of Pure and Applied Logic.

6. Making the supercompactness of $\kappa$ indestructible under $\kappa$-directed closed forcing, Israel Journal of Mathematics 29 (1978), 385-388. MR 0472529 (57:12226)

7. K. McAloon, Consistency results about ordinal definability, Annals of Mathematical Logic 2 (1970/71), no. 4, 449-467. MR0292670(45:1753)

8. William Mitchell, A Note on Hamkins' Approximation Lemma, available at http://www.math . ufl.edu/mitchell, December 2003.

9. Jonas Reitz, The Ground Axiom, to appear in the Journal of Symbolic Logic.

10. The Ground Axiom, Ph.D. thesis, The Graduate Center of the City University of New York, September 2006.

11. W. Hugh Woodin, Recent Developments on Cantor's Continuum Hypothesis, Proceedings of the Continuum in Philosophy and Mathematics, Carlsberg Academy, Copenhagen, November 2004, to appear.

Mathematics Program, The Graduate Center of The City University of New York, 365 Fifth Avenue, New York, New York 10016-and-Department of Mathematics, The College of Staten Island of The City University of New York, 2800 Victory Boulevard, Staten Island, New York 10314

URL: http://jdh.hamkins.org

E-mail address: jhamkins@gc.cuny.edu

Mathematics Program, The Graduate Center of The City University of New York, 365 Fifth Avenue, New York, New York 10016-and-Department of Mathematics, New York City College of Technology of The City University of New York, 300 Jay Street, BRoOKLYN, NEW York 11201

E-mail address: jonasreitz@gmail.com

Department of Mathematics, University of California at Berkeley, Berkeley, CalIFORNIA 94720

E-mail address: woodin@math.berkeley.edu 